associated with cerebral laceration, elevated cerebrospinal pressure, choked disc and focal signs.

The diagnosis was made by cerebral angiography. Suction of hematoma resulted in the improvement of symptoms.

Cases of chronic subdural hematoma were admitted 13 to 100 days after the trauma. Signs and symptoms are those of elevated intracranial pressure and focal lesions. Cerebral angiography readily established the diagnosis. Symptoms were relieved by the removal of hematoma or suction of liquefield hematoma. Prognosis were excellent. It was felt that suction of hematoma may be difficult in cases where hematoma has been organized or has not been yet liquefied. The case of internal carotid occulsion was admitted because of hemiplegia and aphonia six months after trauma. Cerebral angiography established the diagnosis. Administration of vasodilator agent combined with stellate block brought forth the relative relief. Early diagnosis should result in a better improvement. In arteriovenous fistula, no pulsating exophthalmos was present. No occular palsy was evident. Convulsion was not seen. Ligature of common carotid artery resulted in complete relief of the symptoms.

\title{
15. Pathological Physiology and Treatment in the Acute Stadium of Cerebral Injury.
}

\section{T. Mitsuno}

The 2nd Dept. of Surg., Iwate Med. College

It has been emphasized that hazard due to pressure cone occurred if lumbar puncture and removal of CSF was done in acute stadium of cerebral injury. But lumbar puncture is of benefit and useful for the purpose of diagnosis of cerebral compression and subarachnoid hemorrhage.

Main changes in pathological physiology of acute stadium after cerebral injury are inhibition in respiration and cerebral circulation, especially disturbance of circulation of vertebral artery resulting anoxia of the brain stem.

\section{Clinical Studies on Intracranial Hematomas.}

S. KIKUCHI

Juntendo Univ. School of Med.

1) Clinico-statistical observation

Material: 93 cases of intracranial hematomas which were treated in our clinic and 298 cases of autopsy at Tokyo Medical Examiner Office. 
Epidural hematomas were comparatively few in children. Subdural hematomas were found in any age and in any region though less in occipital. Most cases of epidural hematomas associated with scalp injuries and skull fractures, and occured at the same side of the temporal scalp injuries. From this postmortem examination, it will be concluded that scalp injuries and fractures of the localisation of the hematoma.

Acute subdural hematomas were found in 81.2 percent of them were associated with cerebral contusions and lacerations and other cerebral injuries. From these data it is supporated that the subdural hematoma is likely to occur at the frontal and temporal regions where is the favourite site of the cerebral contusion. 2) Ultrasonic examination

We have applied ultrasono-echography in order to diagnose acute and chronic subdural hematomas. This ultrasonic echogram have two apparatus. Namely, which are A-Scope and B-Scope (Ultrasono-tomography). At this time reported about the application of A-Scope which indicats echoes as the wave pattern on the cathod ray screen. The suitable frequency of ultrasonic pulse wave in these cases are 1-10 megacycle per second. We examine the shift of the 3rd ventricle pressed by subdural hematoma and abnormal echo of subdural hematoma.

This method was applied to the out patients after craniotomy in operating room. Accurate depth and extent of subdural hematoma was determined.

After operation, we usually observe shift of the $3 \mathrm{rd}$ ventricle that gradually return to the midline.

Recently discovered that ultrasonic attenuation was related to the brain edema as a rule. This examination is very valuable for the diagnosis and to determine the operative results.

\title{
17. Experimental and Clinical Studies of Brain Injuries.
}

\section{S. ENDO}

\author{
Surg. Dept. of Fukushima Med. College
}

1) Influence of brain injuries to endocrine system

In rabbits with experimental brain injuries, the fluctuation of neurosecretion was histologically examined at the pituitary posterior lobe and hypothalamus, employing the Gomori-Halmi Staining method. In cases with general brain injuries without the hypothalamic lesions, Gomoriphyl substances increased one hour after the injuries, accompanying the gradual decrease and then recovered to the normal state in about one week. In cases with hypothalamic lesions the decrease of neurosecretion was observed several hours after the trauma but about 48 to 72 hours the abnormal intense increase, regarding as a rebound phenomen, was temporary recognized. 